\title{
A simulated measurement for COVID-19 pandemic using the effective reproductive number on an empirical portion of population: epidemiological models
}

\author{
Belal Alsinglawi ${ }^{1} \cdot$ Omar Mubin $^{1} \cdot$ Fady Alnajjar $^{2} \cdot$ Khalid Kheirallah $^{3} \cdot$ Mahmoud Elkhodr $^{4} \cdot$ \\ Mohammed Al Zobbi ${ }^{1}$ Mauricio Novoa ${ }^{5} \cdot$ Mudassar Arsalan $^{1} \cdot$ Tahmina Nasrin Poly $^{6} \cdot$ Munkhjargal Gochoo $^{2}$. \\ Gulfaraz $\operatorname{Khan}^{7} \cdot$ Kapal Dev ${ }^{8}$
}

Received: 7 May 2021 / Accepted: 21 September 2021

(C) The Author(s), under exclusive licence to Springer-Verlag London Ltd., part of Springer Nature 2021

\begin{abstract}
COVID-19 as a global pandemic has had an unprecedented impact on the entire world. Projecting the future spread of the virus in relation to its characteristics for a specific suite of countries against a temporal trend can provide public health guidance to governments and organizations. Therefore, this paper presented an epidemiological comparison of the traditional SEIR model with an extended and modified version of the same model by splitting the infected compartment into asymptomatic mild and symptomatic severe. We then exposed our derived layered model into two distinct case studies with variations in mitigation strategies and non-pharmaceutical interventions (NPIs) as a matter of benchmarking and comparison. We focused on exploring the United Arab Emirates (a small yet urban centre (where clear sequential stages NPIs were implemented). Further, we concentrated on extending the models by utilizing the effective reproductive number $\left(R_{\mathrm{t}}\right)$ estimated against time, a more realistic than the static $R_{0}$, to assess the potential impact of NPIs within each case study. Compared to the traditional SEIR model, the results supported the modified model as being more sensitive in terms of peaks of simulated cases and flattening determinations.
\end{abstract}

Keywords COVID-19 $\cdot$ Simulation $\cdot$ SEIR $\cdot$ Epidemiologic methods $\cdot$ Outbreak $\cdot$ Effective reproductive number

Belal Alsinglawi and Omar Mubin are joint first authors.

Belal Alsinglawi

b.alsinglawi@westernsydney.edu.au

Omar Mubin

o.mubin@westernsydney.edu.au

Fady Alnajjar

fady.alnajjar@uaeu.ac.ae

1 School of Computer, Data and Mathematical Sciences, Western Sydney University, Rydalmere, NSW 2116, Australia

2 College of Information Technology, United Arab Emirates University, Al Ain, UAE

3 Department of Public Health, Medical School of Jordan University of Science and Technology, Irbid, Jordan

4 School of Engineering and Technology, Central Queensland University, Rockhampton, Queensland, Australia
5 School of Built Environment, Western Sydney University, Rydalmere, NSW 2116, Australia

6 College of Medical Science and Technology, Taipei Medical University, Taipei 101, Taiwan

7 College of Medicine and Health Sciences, United Arab Emirates University, Al Ain, UAE

8 Department of Institute of Intelligent Systems, University of Johannesburg, Johannesburg, South Africa 


\section{Introduction}

Starting in 2020, humankind has increasingly suffered from the spread of a new pandemic characterized by acute respiratory and vascular symptoms produced by a novel coronavirus strain known as SARS-CoV-2 [1]. The virus, which initially emerged in Wuhan, China, in November 2019 later was considered a full-fledged outbreak before being declared by the World Health Organisation (WHO) as a public health emergency of international concern [2] around early 2020. Today, COVID-19 has no known approved vaccine, and no treatment is considered effective. Meanwhile, governments and health institutions need assistance to visualize, simulate and assess effective Nonpharmaceutical interventions ((NPIs) to mitigate this virus' unpredictable behaviour and control its spread. Modelling techniques allow simulation and prediction of Covid-19 growth trends and guide pre-emption and preparation. However, it is important to properly introduce model parameters to understand the spread pattern of the infection under different mitigation strategies [3]. NPIs utilized to mitigate the spread of the COVID-19, such as lockdown strategies, have served as effective input to the simulation and allowed to present a range of multiple output scenarios. We have also observed a range of data mining as well as statistical and mathematical approaches. The Susceptible Exposed Infectious Recovered (SEIR) model is a widely used mathematical technique to evaluate mitigation strategies and NPI measures [4]. The SEIR model relies on various disease outbreak parameters, which the scientific community understands much better now than at the earlier stage of the COVID-19 pandemic.

Furthermore, the model represents various categories of symptomatic levels, providing a more accurate simulation of the pandemic. Prior work in mathematical modelling has shown the implementation of SEIR for specific regions and its ability to be modified to model-specific research aims or scenarios, such as in [5, 6]. In work presented in [7], the authors discussed a range of parameters that can be introduced to model COVID-19 and improve the accuracy of SEIR models, as applied to eight countries. We also noted the explicit application of SEIR modelling to specific countries. As an example, in [8], for the case of China, a layer of quarantined patients was incorporated, as well as those who had passed away, ultimately allowing a prediction of peaks in various regions of China. In [31], the SEIR model was modified to include domestic passenger movement data to predict the epidemic's peak. In [9], the conventional SEIR model was applied to various social distancing mitigation strategies, where the sustained application of NPIs was able to mitigate the spread of COVID-19 infection. SEIR modelling was also applied to project the health infrastructure needs, such as ICU beds and hospitalization needs, in France [10].

In this work, we report on how the effect of NPI measures can be investigated and compared according to the change of effective reproductive number $\left(R_{\mathrm{t}}\right)$ using simulation techniques. A case study was selected for simulation from the United Arab Emirates (UAE). The UAE case represents a growing urban centre with a highly social and mobile society that has slowly exited its lockdown strategies after an initial outbreak of COVID-19 [11, 12]. It is also a multi-cultural nation with a diverse diaspora, two major air transport hubs and a high standard of living. Therefore, the UAE deserves individual focus concerning the potential spread of COVID-19 since it can provide valuable insight to other similar countries. We acknowledge that previous work already discussed NPI measures undertaken by South Asian and Gulf countries to mitigate the spread of COVID-19. However, those lack meaningful modelling results [13].

In sum, the case study of the United Arab Emirates was chosen due to the following clear differential aspects. UAE had gradually relaxed its lockdown strategies (such as reopening of international flights as soon as possible). Secondly, most of the interventions for UAE were not publicly available in a clear chronological form to the authors, for example, via the governmental web-sites. Recent research reported that the primary information source for health care workers in the UAE is social media [14] and not authentic governmental sources [15]. We note that the utilization of the UAE case study allows us to judge the suitability and sensitivity of our proposed model to capture intervention settings and scenarios. The application of these simulation models was further considered by the availability of information regarding mitigation strategies for each country. As stated in the case of UAE, no clear or segmented mitigation strategies were available to the authorship team to guide the model simulation inputs. As such, this provided further evidence of the impact of disclosing and inputting mitigation strategies on simulating COVID-19 spread within different populations. To reiterate, our study aims to provide future forecasting estimations about the spread of COVID-19 in the UAE with different scenarios using the SEIR models. Specifically, we introduce two additional layers by splitting infectious into asymptomatic/symptomatic mild and symptomatic severe. This discretion is integral for the study of COVID-19 spread because it is established that many patients can go untested due to no visible symptoms. However, the virus can transmit from such patients and many patients diagnosed show mild symptoms [16]. 


\section{Methods}

A country-based case study was simulated in the current research. The UAE case study evaluated the effects of NPIs when clear policies are neither publicly communicated nor publicly available.

\subsection{Model description and parameters}

A simulated compartmental model was implemented to measure the spread of COVID-19 using an empirical population sample (across both case studies). Our simulated model is built upon an extended version of the SEIR Model [17]. We have used a portion of the UAE population $(2,998,325)$ which is the total empirical population sample in GleamViz software in this study into 5 compartmental state: Susceptible $(S)$, Exposed $(E)$, Infected Asymptomatic-mild $\left(I_{a}\right)$, Infected Symptomatic Severe $\left(I_{\mathrm{s}}\right)$ and Recovered $(R)$. The constant $N\left(N=S+E+I_{a}+I_{\mathrm{s}-}\right.$ $+R)$ denotes the total population $(N=2,998,325$ for UAE (S1). The categories of the compartments are further described below.

- Susceptible (S) All non-immune susceptible empirical population samples in our study.

- Exposed (E) latent but not yet infectious or "have no symptoms, and they cannot spread the virus yet".

- Infected asymptomatic-symptomatic mild $\left(I_{a}\right)$ Refers to transmission of the virus from a person who does not develop symptoms or with mild symptoms [18] to another person (not yet latent but suspected).

- Infected symptomatic severe $\left(I_{s}\right)$ The state of COVID19 infection can progress to severe disease with dyspnoea and severe chest symptoms [19].

- Recovered $(R)$ Population showing immunity for COVID-19 after infection recovery.

In the course of many diseases, there are an unknown fraction of the in-infected hosts that are still able to spread the disease while remaining symptoms-free (asymptomatic) [20]. In our model, asymptomatic cases are combined with mild cases in the same fraction. This confirms the reported proportion of the infections according to WHO [18]. Rationally, splitting the asymptomatic and mild into $I_{\mathrm{a}}$ compartmental state and the symptomatic severe into Is compartmental state and the understanding of spreading growth for each compartment are a worthwhile attempt. Further, it is deemed as an important research task to evaluate the behaviour of each compartment in the pandemic event and for further compartments evaluation in relation to the NPI mitigation strategies such as social distancing, lockdown, wearing masks, and more strategies arise as the pandemic progress (which reinforces our aim to apply the modelled simulation to two distinct case studies). Therefore, our proposed epidemiological model is espousing the asymptomatic mild and severe states according to the WHO new classification [18] for COVID19 infected cases. The (beta) time-based(t) $\left(\beta_{\mathrm{t}}\right)$ describes the transmission rate (vary according to social distancing, remote working, closing schools, wearing masks, etc.). Alpha $(\alpha)$ indicates the reduction in the transmission rate of $\beta$ in the isolated infectious symptomatic (severe), where patients are isolated [21]. The incubation period $(\gamma)$ is a period from the state of the exposure to the disease to become in-infectious. Our model used the value of $(\gamma=1 /$ 5.2 'days') [22]. The recovery rate $(\mu)$ in our model indicates the time until an infectious case is recovered. Previous research [12] tells us the recovery time for COVID-19 is 14 days. We have used this value (recovery rate is ( $\mu=1 / 14$ days) in our model. More information regarding the parameters used in our study is discussed in Table 1. The COVID-19 pandemic transmission in our model can be described by:

$\dot{S}=-\beta_{\mathrm{t}} S\left(I_{\mathrm{a}}+I_{\mathrm{s}}\right)$

$\dot{E}=\beta_{\mathrm{t}} S\left(I_{\mathrm{a}}+I_{\mathrm{s}}\right)-\gamma E$

$\dot{I_{\mathrm{a}}}=\gamma P_{\mathrm{a}} E-\mu I_{\mathrm{a}}$

$\dot{I_{\mathrm{s}}}=\gamma\left(1-P_{\mathrm{a}}\right) E-\mu I_{\mathrm{s}}$

$\dot{R}=\mu I_{\mathrm{a}}+\mu I_{\mathrm{s}}$

where $N=S+E+I_{\mathrm{a}}+I_{\mathrm{s}}+R$. We have calculated the $\operatorname{beta}_{(\mathrm{t})}\left(\beta_{\mathrm{t}}\right)$ according to equation (6):

$\beta_{\mathrm{t}}=R_{\mathrm{t}} \mu / P_{\mathrm{a}} \alpha+\left(1-P_{\mathrm{a}}\right)$

We proposed model on the basis of SEIR Model [4] (Fig. 1). The traditional SEIR model equation is formed as the following:

$\dot{S}=-\beta t S I$

$\dot{E}=\beta t S I-\gamma E$

$\dot{I}=\gamma E-\mu I$

$\dot{R}=\mu I$

where $N=S+E+I+R$. Figure 1 represents the traditional compartments for the SEIR model. The $\operatorname{beta}_{(\mathrm{t})}\left(\beta_{\mathrm{t}}\right)$ is time-dependent. Therefore, $\left(\beta_{\mathrm{t}}\right)$ is denoted with the following equation:

$\beta_{\mathrm{t}}=R_{\mathrm{t}} \mu$

Figures 1 and 2 compare the dissimilarity between $\left(I_{\mathrm{a}}\right.$ and $I_{\mathrm{S}}$ ) compartments of (Fig. 2) to the infected compartment (I) in Fig. 1. Equations (1-6) are the COVID-19 transmission equations of (Fig. 2) in contrast with SEIR Eqs. (7-11). 
Table 1 Model parameters and description

\begin{tabular}{|c|c|c|}
\hline Parameter & Description & Value(s) and Ref \\
\hline$\gamma$ & $\begin{array}{l}\text { The incubation period from the state of exposure to the disease to become } \\
\text { infectious for } \mathrm{S} 1\end{array}$ & 5.2 days $[22]$ \\
\hline $\mathrm{Pa}$ & Probability of being asymptomatic-symptomatic (Mild) & $0.8[18]$ \\
\hline Ps & Probability of being severe symptomatic requiring hospitalization $=(1-P a)$ & $0.2[18]$ \\
\hline$\beta_{\mathrm{t}}$ & $\operatorname{Beta}(t)$ or transmission rate describes the spread of disease in the community & $\begin{array}{l}\text { Equation } 6 \text { for S1 and Eq. } 11 \text { for S1 (given } \\
\text { above) }\end{array}$ \\
\hline$\alpha$ & Alpha: reduction in transmission rate (severe) & $0.5[21]$ \\
\hline$\mu$ & $\begin{array}{l}\text { The recovery rate for }(\mathrm{S} 1) \text { : Indicates the time until the infectious case becomes re- } \\
\text { covered }\end{array}$ & $\begin{array}{l}14 \text { days }[12,23] \mu(1 / 14 \text { days }=0.07 \\
\text { "day }^{-1} \text { ") }\end{array}$ \\
\hline
\end{tabular}

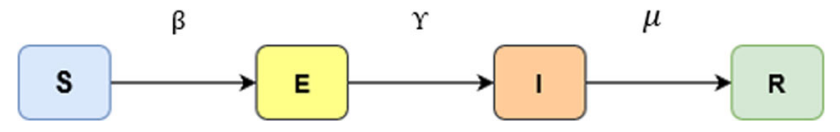

Fig. 1 SEIR model

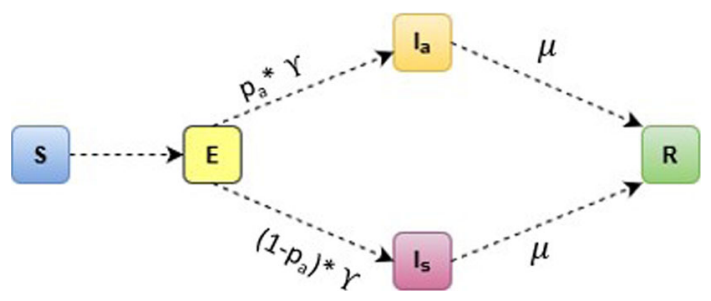

Fig. 2 Architecture of the proposed $\mathrm{SEI}_{a} \mathrm{I}_{s} \mathrm{R}$ model (S1)

\subsection{Estimating the effective reproductive number}

The $R_{\mathrm{t}}$ (effective reproductive number) measures the transmission potential of COVID-19, which is also referred to as the average number of people who will catch the disease from a single infected individual. When the pandemic occurs, the effective reproductive number $R_{\mathrm{t}}$ measures which will become in-infected per infectious person at a time (t). The most well-known version of $R_{\mathrm{t}}$ is the basic reproductive number $R_{0}$. However, the $R_{0}$ is a single measure that does not reflect changes in disease transmission, behaviours and restrictions in communities over time. Alternatively, as the pandemic progresses, mitigation strategies could be tightened, more restrictions imposed, or relaxed. This enables $R_{\mathrm{t}}$ to vary over time. Therefore, the $R_{\mathrm{t}}$ value is subject to variation after or before the introduction of NPIs. To estimate the $R_{\mathrm{t}}$, we have used a real-time Bayesian estimation [24] and implementation of work by [25]. Figure 3 in the results and discussion section provides the calculated $R_{\mathrm{t}}$ values for UAE with a value of 2.4 . Modelling software we have utilized is GLEAMviz client simulator [26], which combines world data such as

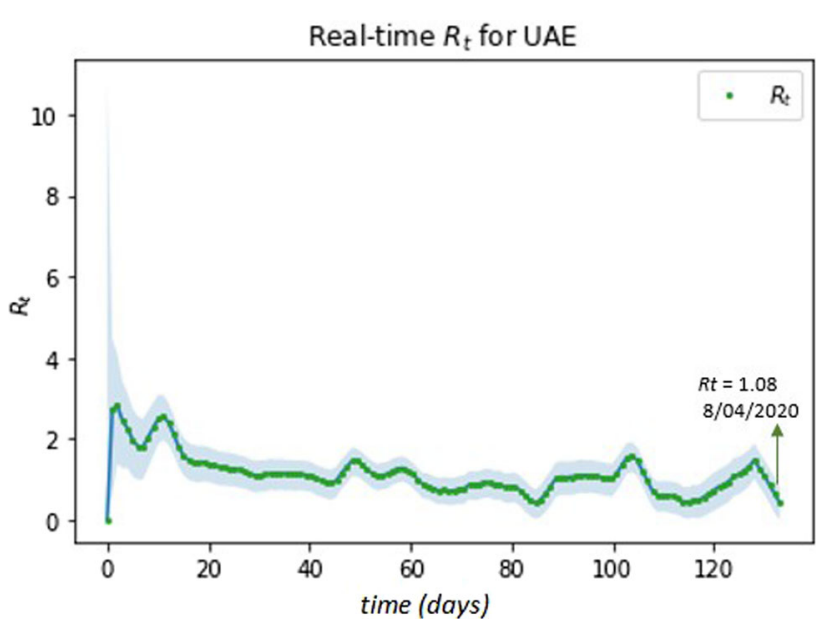

Fig. 3 Real-time $R_{\mathrm{t}}$ for UAE from $23 / 3 / 2020$ to $2 / 8 / 2020$

countries populations and human mobility. The GLEAMviz simulator elaborates compartmental stochastic models [27] for disease transmission in a global epidemic event. To forecast the number of estimated future compartments for the COVID-19 epidemic in the UAE, we have exploited a previous model, "Global Epidemic and Mobility GLEaM H1N1 schematic" [26] depicts the spread of such as an epidemic disease. We considerably modified the model to include the compartment of asymptomatic mild and symptomatic severe layers. Noting that $I_{\mathrm{a}}$ represents the asymptomatic mild cohort and the $I_{\mathrm{s}}$ represents the symptomatic severe cohort in the study. Figure 2 represents the schematic for our proposed epidemiological model compartments.

\section{Results and discussion}

Currently, there is no cure or effective vaccine for Covid19 while the pandemic continues to spread, and there are more daily confirmed positive cases and deaths recorded 
worldwide. Aptly, it is necessary to maintain and measure NPIs effectiveness and figure out how to flatten the pandemic curve with long term interventions until the time that successful vaccines are widely available or effective treatment is available. This section reflects on findings from the UAE case study, particularly where detailed NPIs are not publicly available. Our model is sensitive to the contact rate that determines the change of the $R_{\mathrm{t}}$ value, which is the essential entry to our simulation to reflect the policy outcomes in real-time $R_{\mathrm{t}}$ measurement. The GLEAMviz simulation was run to initialize the spread of COVID-19 in the UAE starting on the 29th of January 2020. Since the GleamViz is limited to 365 days, our simulation ends on 28/1/2021 for UAE. Then, we simulated our proposed model $S E I_{\mathrm{a}} I_{\mathrm{S}} R$ (S1) for the situation of UAE. After that, we demonstrated the results of the $\mathrm{S} 1$ simulation on an empirical portion of the population of the UAE. The $\mathrm{S} 1$ simulation considered the changes in the $R_{\mathrm{t}}$ according to the changes (tightening or easing) in policies in UAE, between $29 / 1 / 2020$ and $2 / 8 / 2020$. The data used to calculate the $R_{\mathrm{t}}$ were fetched from the Github repository of "Our World in Data" [28] for the UAE case. Furthermore, the data preprocessing step was applied to get the daily new cases from the John Hopkins official Github. The data attributes are the date, name of the country and the number of new daily cases $(\mathrm{k})$ for the UAE. This step is essential in measuring $R_{\mathrm{t}}[24,25]$. We have decided to change $R_{\mathrm{t}}$ for simulation inputs by 0.5 points of $R_{\mathrm{t}}$ each increase in the $R_{\mathrm{t}}$ or increment. This is assuming the 0.5 value has a noticeable impact on the simulation results.

\subsection{COVID-19 simulation in undisclosed public health strategy for the public (UAE)}

Figure 3 shows the real-time $R_{\mathrm{t}}$ for UAE. Since UAE went through different levels of social distancing, restrictions and easing of restrictions strategies, estimating the $R_{\mathrm{t}}$ is an essential task to measure and reflect the policy outcomes on empirical data of (Fig. 2) over time. The simulation parameters values (Table 1) are the input for extended SEIR (Fig. 2) simulation. Our strategy was to update the extended SEIR upon each 0.5 difference in $R_{\mathrm{t}}$ value to adopt the changes of a policy at a point of time. We have fed the model with $R_{\mathrm{t}}$ values in the GleamViz's exception layer, indicating the policy measured at a time (t). Since we were limited to the new COVID-19 daily cases obtained by the Github repository of Our World in Data [2], the $R_{\mathrm{t}}$ measure started on 23/3/2020. However, we kept the value of $R_{0}$ constant from 29/1/2020 until 23/3/2020. We assumed that the $R_{0}=2.5$ in our model based on information from the WHO [18] since there was no available data about $R_{\mathrm{t}}$ at the beginning of the pandemic.
$R_{\mathrm{t}}$ in the UAE fluctuated between zero at the beginning of the pandemic and 3 , during the first two weeks of the pandemic. After that, a decline in the $R_{\mathrm{t}}$ was noted, reaching around one around the 40th day. Around the 50th day of the pandemic, $R_{\mathrm{t}}$ increased to around 2 and declined after that to less than one between the 60th and 90th day of the pandemic. The sharpest decrease in $R_{\mathrm{t}}$ was observed between day 110 and 120 of the pandemic. By the end of the simulation, $R_{\mathrm{t}}$ was noted as 1.08 .

As of 23/05/2020, the median rate $(95 \%$ CI) of asymptomatic mild cases was $11.25(5.62-12.26)$ per1000 population with a cumulative median of 356.64 (173.07-540.17) per 1000 population (Fig. 4a). In the severe COVID-19, cases peaked by $20 / 5 / 2020$ and the simulation predicted that there will be no severe cases after $16 / 12 / 2020$. The median rate was simulated at 2.83 (1.35-3.02) per 1000 population and a cumulative median of 81.36 (39.02-126.22) per 1000 population, as shown in Fig. 5b. Simulated severe COVID-19 cases are essential to estimate the population that may require advanced health services, critical care services or even hospitalization care. Simulation of severe cases will facilitate estimating the needs for health services and identifying anticipated needs for patients with the severe diagnosis. After that, a simple comparison of estimated numbers and availability of health services will provide a valuable need assessment and identify potential gaps in medical services. However, the lack of healthcare indicators from the UAE limited such comparison and restricted our abilities to anticipate the gap as mentioned above.

Recovered cases in S1 followed the same trends of Asymptomatic-mild and severe cases. Figure $4 \mathrm{c}$ illustrates that the peak median recovered cases reached 12.98 per 1000 (6.92-13.41), and median Cumulative was 418.15(206.55-622.50) per 1000 population, as shown in Fig. 4c. The simulation, according (Fig. 2), estimated recovered cases to flattened by 27/1/2021. With reference to the flattening of the curve, in general, we observed that our results are in line with prior literature [29], where it was shown that lockdown and stringency measures are required to be sustained for anywhere between 3 and 5 months to flatten the curve (albeit for the case of UK). With the increased global concerns of COVID-19, strict NPI measures become necessary to mitigate the risks associated with COVID-19. Citizens' commitment is critical to control the epidemic.When citizens adapt to the NPI measures, a reduction in the spread of the epidemic is expected. The combined efforts from both governments and citizens are then critical for designing and adapting effective NPI measures. This is reflected in the epidemic curve of the pandemic. In the current study, the effect of NPIs was assessed utilizing $R_{\mathrm{t}}$ using advanced simulation models for UAE. The model established potential evidence of 
(Median, Lower $95 \% \mathrm{Cl}$, Upper $95 \% \mathrm{Cl}$ )for

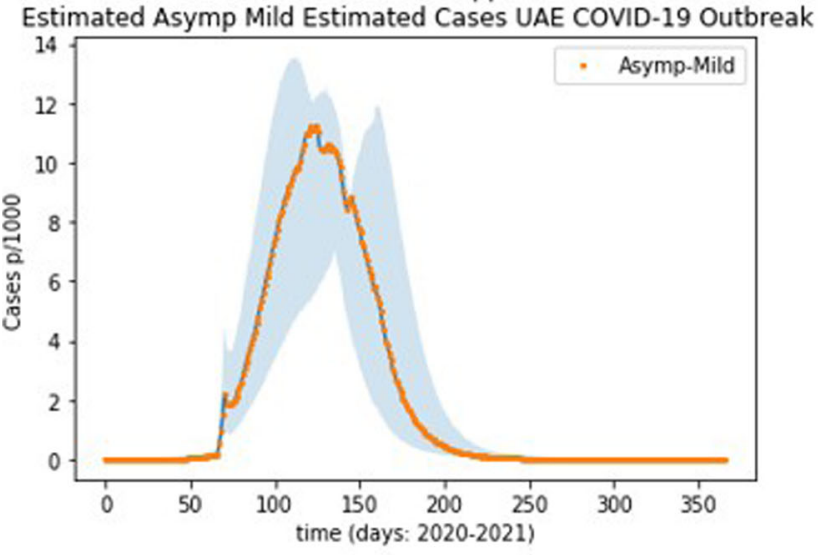

(a)

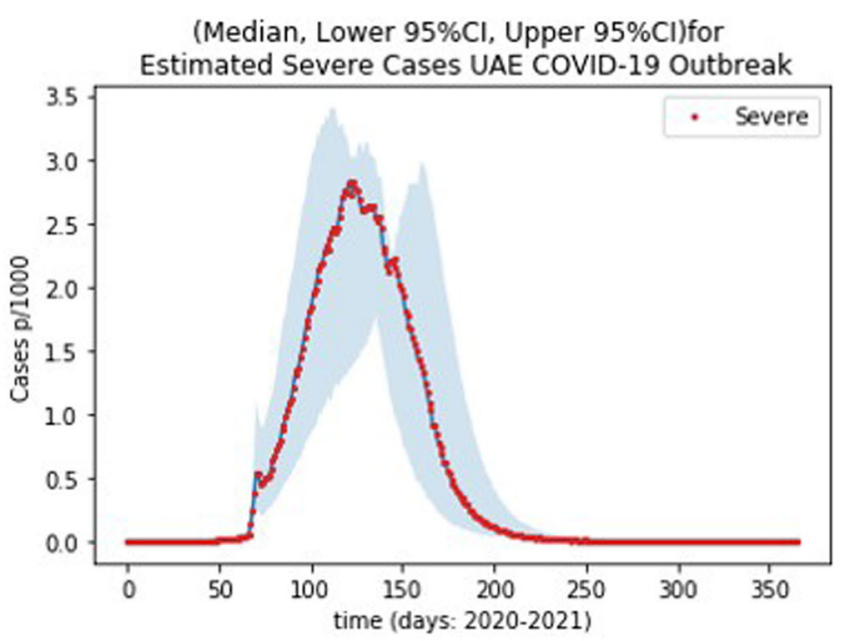

(b)

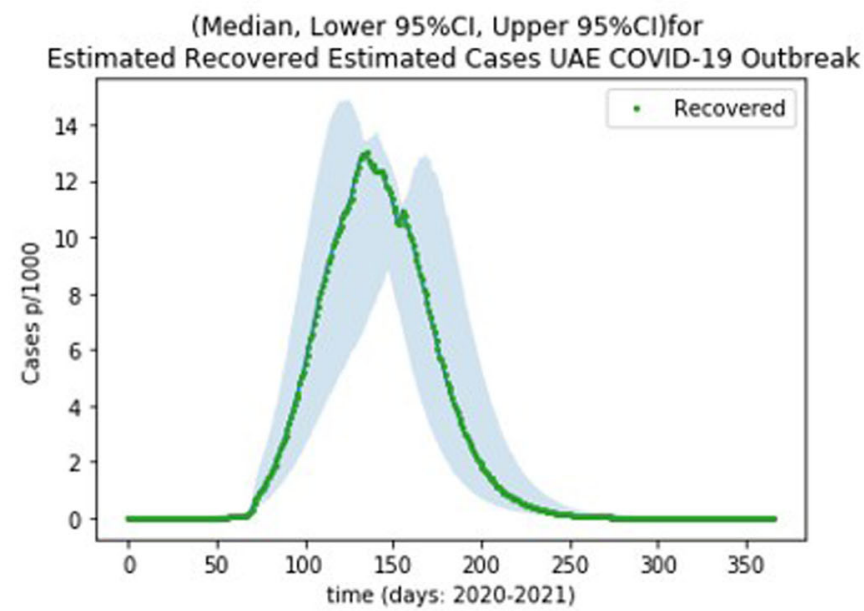

(c)

Fig. 4 a-c Median, lower 95\%CI, upper 95\%CI) for asymptomatic mild, severe and recovered estimated cases (S1)

effective NPIs to control the spread of COVID-19, especially when model modifications were introduced to meet the characteristics of the pandemic. Adopted NPIs in UAE geographic locations effectively reduced the effective reproduction number below one. Further, the results indicated that the rapid introduction of NPIs has a more effective reduction in the spread of the epidemic.Multiple models evaluated the effectiveness of state measures to control COVID-19 spread. A direct link was established between the effectiveness of NPIs [11] on reducing the reproduction rate $\left(\mathrm{R}_{t}\right)$. Our results are in line with the literature. They indicate that effective implementation of NPI measures has potentially profound consequences on the epidemic curve of COVID-19 by reducing the number of newly reported or simulated cases and reducing the effective reproduction number. The message behind such results is a cornerstone for communicating public health policies and the implementation of NPIs. The sensitivity of $R_{\mathrm{t}}$ to the contact rate is critical in spreading or containing the spread of COVID-19. The effect of contact rates is, in turn, dependent on NPIs, which then are critical for mitigating the disease. Regardless, our study provides evidence that the effect of NPI measures could be evaluated and discussed using the effective reproductive rate. This is an added value to public health professionals and could be used when designing and implementing mitigation strategies, such as discussing whether suppression or control is more appropriate. On the other hand, traditional SEIR models seem to be limited in assessing the effect of NPI measures on the epidemic curves of COVID-19 [30]. This note is directly related to the need to consider the characteristics of the disease, with COVID-19 representing itself uniquely as asymptomatic cases that needed to be finetuned when designing the model compartments. Disease severity, therefore, is deemed critical for modelling and simulating the transmission of COVID-19 within 


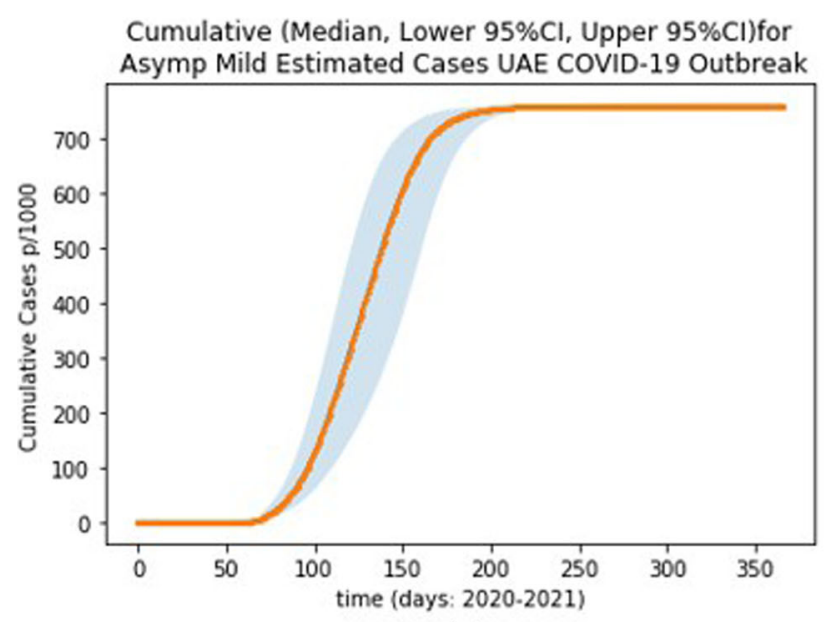

(a)

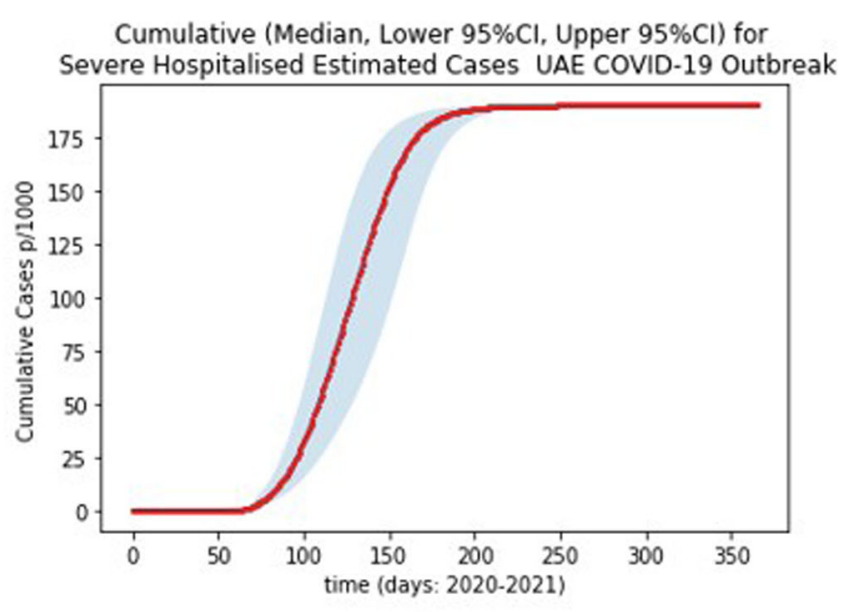

(b)

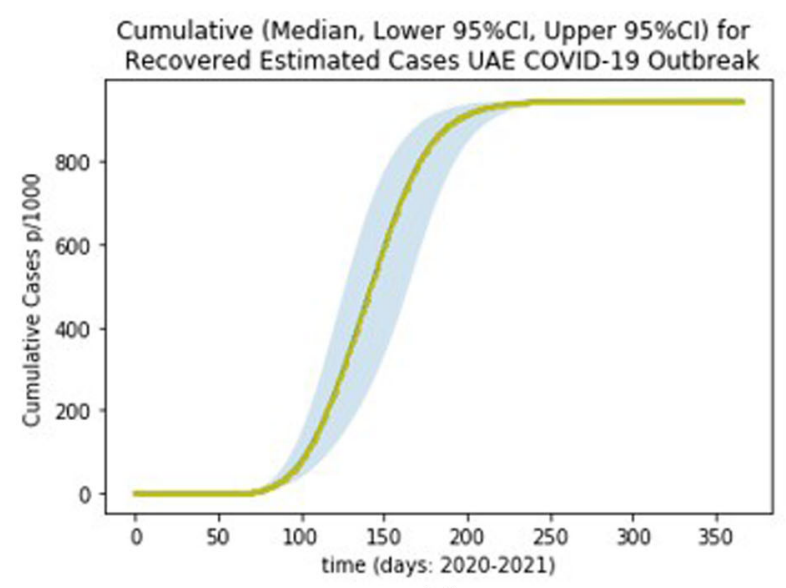

(c)

Fig. 5 a-c Cumulative (median, lower 95\%CI, upper 95\%CI) for asymptomatic-mild, severe and recovered estimated cases (S1)

populations. Within the uncertainties associated with COVID-19, time will tell if these asymptomatic mild cases are of more significant concern for disease transmission. Regardless, modelling and simulation techniques should consider modifying the traditional SEIR to present the epidemic curve better. A vital research implication of our work is that estimating the proportion of severe cases requiring hospitalization using SEIR extended epidemiological models may help healthcare decision-makers during the pandemics. For instance, hospital healthcare decisionmakers such as beds managers, clinicians, and healthcare managers can work more effectively and plan the beds and staffing. Moreover, the healthcare assessments systems such as the clinical decision systems to predict patients' length of stays in ICU or COVID-intensive care units, including ventilators, are utilized. This is an essential task for hospitals to manage beds scarcity, especially in uncertainties such as pandemics.

\section{Study limitations}

Our study provides an interesting outlook on the computation of $R_{\mathrm{t}}$ concerning stated interventions; however, there are some limitations also associated with the simulation. Our model is evaluated on empirical population data. We did not examine our model on real confirmed cases due to the lack of many variables necessary for stochastic compartment models. Transmission data may simply not be available or is made private by the authorities, which has ultimately limited our potential to run the model on realworld data and evaluate the predictions of the simulated model against (Asymptomatic-Mild) and real severe cases. We have also assumed that the entire population of the sample country (in our case UAE) is susceptible. Prior work has utilized other ranges, such as 70\% [23]. Further, we have not executed any complementary logistic modelling on our scenarios for the UAE.Furthermore, we did not study and report the severe cases that require 
hospitalizations. Therefore, a future study should include forecasting severe cases that may require hospitalizations in the model. Finally, the Glemaviz software application does not allow accessing the mathematical equations used to run the model. This limits our abilities to adjust disease characteristics within the equations. This may be a reason behind discrepancies in S1 and SEIR models. Still, Glemaviz is a user-friendly application that allows public health professionals to run simulation models without an in-depth understanding of advanced mathematical equations.

\section{Conclusions}

Our study attempted to extend the SEIR model by forking the infectious compartment into two subcategories, namely asymptomatic mild or symptomatic severe. We have also illustrated how the effective reproductive number (and its change over time) can be computed using available parameters, despite the lack of realistic data. This computation has allowed us to forecast and predict the outlook of COVID-19 in the UAE as our sample country of an investigation by using the two variations in the SEIR model. Our results show us that the modified SEIR model is more sensitive and can also determine when the diffusion will flatten.We also summarize certain limitations of our modelling; the most concerning is the lack of real empirical data. Nevertheless, with the comfort of prior literature [31] where it is highlighted that the SEIR modelling is appropriate for longer-term projections, and it provides a range of parameters for specific contexts, we ascertain that our determinations can drive public health policy in small to mid-size countries such as the UAE. In future work, we will predict hospital length of stay for COVID-19 inpatients admitted into hospital departments such as intensive care unit (ICU) by using the advancement of machine learning models [32] and deep neural networks models [33] to assist hospital beds managers and clinical practitioners with resources utilizations during the time of uncertainties such as pandemic.

Funding Not applicable.

Availability of data and material The simulation and the algorithm are available upon request. Please contact the first author of this study, Belal Alsinglawi b.alsinglawi@westernsydney.edu.au; b.alsinglawi@gmail.com.

\section{Declarations}

Conflict of interest The authors declare that they have no conflict of interest.

\section{References}

1. Ackermann M, Verleden SE, Kuehnel M, Haverich A, Welte T, Laenger F, Vanstapel A, Werlein C, Stark H, Tzankov A (2020) Pulmonary vascular endothelialitis, thrombosis, and angiogenesis in covid-19. New Engl J Med 383:120-128

2. Brahma D, Chakraborty S, Menokee A (2020) The early days of a global pandemic: A timeline of covid-19 spread and government interventions. Brookings, Washington, DC

3. Lai S, Ruktanonchai NW, Zhou L, Prosper O, Luo W, Floyd JR, Wesolowski A, Zhang C, Du X, Yu H (2020) Effect of nonpharmaceutical interventions for containing the covid-19 outbreak: an observational and modelling study. medRxiv

4. Li MY, Graef JR, Wang L, Karsai J (1999) Global dynamics of a Seir model with varying total population size. Math Biosci 160(2):191-213

5. Dil S, Dil N, Maken ZH (2020) Covid-19 trends and forecast in the eastern mediterranean region with a particular focus on Pakistan. Cureus 12(6): 8582

6. Farooq J, Bazaz MA (2020) A novel adaptive deep learning model of covid-19 with focus on mortality reduction strategies. Chaos Solitons Fractals 138:110148

7. Zhou X, Ma X, Hong N, Su L, Ma Y, He J, Jiang H, Liu C, Shan G, Zhu W (2020) Forecasting the worldwide spread of covid-19 based on logistic model and seir model. medRxiv

8. Peng L, Yang W, Zhang D, Zhuge C, Hong L (2020) Epidemic analysis of covid-19 in china by dynamical modeling

9. Prem K, Liu Y, Russell TW, Kucharski AJ, Eggo RM, Davies N, Flasche S, Clifford S, Pearson CA, Munday JD (2020) The effect of control strategies to reduce social mixing on outcomes of the covid-19 epidemic in Wuhan, China: a modelling study. The Lancet Public Health 5:e261-e270

10. Massonnaud C, Roux J, Crépey P (2020) Covid-19: forecasting short term hospital needs in France. medRxiv

11. AlQutob R, Moonesar IA, Tarawneh MR, Al Nsour M, Khader Y (2020) Public health strategies for the gradual lifting of the public sector lockdown in Jordan and the United Arab Emirates during the covid-19 crisis. JMIR Public Health Surveill 6(3):e20478

12. Tang B, Bragazzi NL, Li Q, Tang S, Xiao Y, Wu J (2020) An updated estimation of the risk of transmission of the novel coronavirus (2019-ncov). Infectious Disease Model 5:248-255

13. Bloukh SH, Shaikh A, Pathan HM, Edis Z (2020) Prevalence of covid-19: A look behind the scenes from the UAE and India

14. Bhagavathula AS, Aldhaleei WA, Rahmani J, Mahabadi MA, Bandari DK (2020) Novel coronavirus (covid-19) knowledge and perceptions: a survey on healthcare workers. MedRxiv

15. Elkhodr M, Mubin O, Iftikhar Z, Masood M, Alsinglawi B, Shahid S, Alnajjar F (2021) Technology, privacy, and user opinions of covid-19 mobile apps for contact tracing: Systematic search and content analysis. J Med Internet Res 23(2):e23467. https://doi.org/10.2196/23467

16. Cascella M, Rajnik M, Cuomo A, Dulebohn SC, Di Napoli R (2020) Features, evaluation and treatment coronavirus (COVID19). StatPearls Publishing, Treasure Island

17. Li MY, Muldowney JS (1995) Global stability for the Seir model in epidemiology. Math Biosci 125(2):155-164

18. Organization WH (2020) Coronavirus disease 2019 (covid-19). Report (2020). https://www.who.int/docs/default-source/cor onaviruse/situation-reports/20200306-sitrep-46-covid-19. pdf?sfvrsn=96b04adf4

19. Velavan TP, Meyer CG (2020) The covid-19 epidemic. Trop Med Int Health 25(3):278-280

20. Chisholm RH, Campbell PT, Wu Y, Tong SY, McVernon J, Geard N (2018) Implications of asymptomatic carriers for 
infectious disease transmission and control. R Soc Open Sci 5(2): 172341

21. Verity R, Okell LC, Dorigatti I, Winskill P, Whittaker C, Imai N, Cuomo-Dannenburg G, Thompson H, Walker PG, Fu H (2020) Estimates of the severity of coronavirus disease 2019: a modelbased analysis. Lancet Infect Dis 20:669-677

22. Liu Y, Gayle AA, Wilder-Smith A, Rocklöv J (2020) The reproductive number of covid- 19 is higher compared to SARS coronavirus. J Travel Med 27:taaa021

23. Wölfel R, Corman VM, Guggemos W, Seilmaier M, Zange S, Müller MA, Niemeyer D, Jones TC, Vollmar P, Rothe C (2020) Virological assessment of hospitalized patients with covid-2019. Nature 581(7809):465-469

24. Bettencourt LM, Ribeiro RM (2008) Real time Bayesian estimation of the epidemic potential of emerging infectious diseases. PLoS ONE 3(5):e2185

25. (2020) https://www.datacamp.com/community/tutorials/replicat ing-in-r-covid19.

26. Balcan D, Hu H, Goncalves B, Bajardi P, Poletto C, Ramasco JJ, Paolotti D, Perra N, Tizzoni M, Van den Broeck W (2009) Seasonal transmission potential and activity peaks of the new influenza a (h1n1): a monte carlo likelihood analysis based on human mobility. BMC Med 7(1):45

27. Greenwood, P.E., Gordillo, L.F.: Stochastic epidemic modeling, pp. 31-52. Springer, Berlin (2009)

28. (2020). https://github.com/owid/covid-19-data/tree/master/pub lic/data
29. Ferguson N, Laydon D, Nedjati Gilani G, Imai N, Ainslie K, Baguelin M, Bhatia S, Boonyasiri A, Cucunuba Perez Z, CuomoDannenburg G (2020) Report 9: impact of non-pharmaceutical interventions (NPIs) to reduce covid19 mortality and healthcare demand. Imperial College Lond 10:491-497

30. Kheirallah KA, Alsinglawi B, Alzoubi A, Saidan MN, Mubin O, Alorjani MS, Mzayek F (2020) The effect of strict state measures on the epidemiologic curve of covid-19infection in the context of a developing country: a simulation from Jordan. Int J Environ Res Public Health 17(18):6530

31. (2020). URL https://www.who.int/news-room/detail/23-01-2020statement-on-the-meeting-of-the-international-health-regulations(2005)-emergency-committee-regarding-the-outbreak-of-novelcoronavirus-(2019-ncov)

32. Alsinglawi B, Alnajjar F, Mubin O, Novoa M, Alorjani M, Karajeh O, Darwish O (2020) Predicting length of stay for cardiovascular hospitalizations in the intensive care unit: Machine learning approach. In: 2020 42nd annual international conference of the IEEE engineering in medicine biology society (EMBC), pp 5442-5445. https://doi.org/10.1109/EMBC44109.2020. 9175889

33. Srinivasu PN, SivaSai JG, Ijaz MF, Bhoi AK, Kim W, Kang JJ (2021) Classification of skin disease using deep learning neural networks with MobileNet V2 and LSTM. Sensors 21(8):2852

Publisher's Note Springer Nature remains neutral with regard to jurisdictional claims in published maps and institutional affiliations. 